\title{
Information technologies at physics practicums in a subject- oriented school
}

\author{
Lyudmila $V$. Tishchenko ${ }^{1, *}$ \\ ${ }^{1}$ Lyceum No.5, Zaraysk, 143062, Moscow region, Russia
}

\begin{abstract}
The paper suggests a new method of implementing an activity approach in a physicsoriented program. Such method implies the use of a system which comprises three types of practical sessions: a laboratory practicum, a practical training aimed at analyzing physical processes on the basis of computerized modeling and a problem-solving practicum. A methodology used for holding practicums provides for an active use of information technologies. At laboratory sessions we use computerized sensors, tablets with an integrated data recorder, an electronic oscilloscope and process data in Excel tables. At practicums aimed at analyzing physical processes, we develop programs in Visual Basic and Pascal in order to visualize physical processes in the form of motion trajectories, dynamic charts; besides, we conduct computer experiments. At problem-solving practicums we use our own PowerPoint "Study Guide for an Interactive Whiteboard", which contains a database of graphic and pictorial physics problems for students of 10-11 grades. Knowledge of information technologies facilitates students' adaptation in modern society, improves education quality.
\end{abstract}

Nowadays we are living in a fast-moving and information-intensive world. Education facilitates the correlation between the needs and abilities of people to understand their environment, enables them to fulfill their potential. As the pace of life becomes faster, such correlation becomes more difficult to establish. Due to the above, modern society gets interested in improving the quality of education - a social category, which determines the state and efficiency of education process. The use of information technologies has a significant impact on the education process itself as well as on its results.

Federal State Education Standards for secondary (complete) general education contains a list of requirements for the study results of physics-oriented programs which stipulates the following: students acquire a skill to formulate hypotheses, test them with experiments, create patterns, study phenomena, describe and explain experiments; independently design and conduct physics experiments (including those, which imply the use of computer technologies); students are able to solve physics problems, choose physics patterns and make assessment calculations based on available data [1].

All these goals are possible to achieve provided that education process in a secondary school is based on an activity approach. Its general idea is as follows: knowledge is obtained and created by students; most of the time students work on their own, the teacher acts as a partner or a consultant. As a way of implementing such activity approach in a subject-oriented school, during physics lessons we use a system, which comprises three types of practicums: a laboratory practicum, a practicum aimed at analyzing physical processes on the basis of computerized modeling and a problem-solving practicum [2]. A lesson plan for all types of physics practicums implies systematic and extensive use of computerized information technologies. Practicums are included into the system of lessons, envisaged by a physics syllabus for a subject-oriented school.

Let us examine in detail peculiarities of practicums, methods of information technologies application at such lessons, influence of the above-mentioned technologies on education process. It should be noted that our developments are of vital importance for secondary schools which do not have modern diversified and specialized laboratories for physics practicums, for regular schools in Russia which represent the vast majority, for physics teachers, who combine different elements of computerized technologies in the course of physics practicums.

Laboratory practicums perform the main function in teaching physics: students independently explore physical phenomena based on the scientific method of perception [3].

Laboratory practicums have the following distinctive features:

1. Students fix the goals of their work at their own discretion.

2. Students independently elaborate a specific work plan.

*Corresponding author: alis11108@yandex.ru 
3. It is required to provide students with "extra" devices in the equipment kit for the work.

4. The work should be done without giving highschool students a detailed instruction, which allows them a wide discretion to suggest their own work methods. This essential difference from a laboratory practicum enables teachers to shape teaching methods: fix the goal, consider the way of reaching it in the course of experimental research.

5. The equipment kit for the work should necessarily include computerized measurement systems and a personal computer.

While organizing laboratory practicums we select the equipment from L-micro kit or any other available equipment. As a rule such kits contain digital and analog measuring instruments. In accordance with the principle of optimal combination of digital, analog and computerized instruments, it is necessary to include computerized sensors in the kit (for instance, sensors of Einstein digital laboratory), a tablet computer with an integrated data recorder, a desktop computer or a laptop.

The use of computerized sensors in the course of physical experiments conducted at laboratory practicums offers a number of benefits for the education process. First, it enables students to measure physical quantities very quickly, with high precision and in any increments, and allows them to take repeated measurements. Second, students may plug in several sensors at a time and measure several parameters of the system simultaneously; besides, the sensors are connected straight to the computer via USB ports with no auxiliary units. Third, the experiment parameters are sent from the sensors to the tablet, where the measuring system is installed (e.g. MiLAB), which automatically processes incoming parameters. The information on the results of the experiment is displayed on the screen in tables, diagrams, graphical curves. Senior students analyze numeric data they receive and calculate necessary quantities. If necessary, pupils may approximate graphical curves of physical quantities, change the scale of axis for plotting charts, observe particular intervals of the chart. Students may copy all the necessary information on the experiment to their computers, save it and process; besides, they may systematize necessary files. If needed the data may be printed out or displayed on an interactive whiteboard.

The above mentioned benefits of an automatic system of processing data of a physical experiment are at the same time the problems of their use. When using sensors, many operations are conducted automatically, senior students do not train such important skills as ability to work with formulas, plot charts, estimate inaccuracies, etc., they mostly act as users of off-theshelf information technologies but not their developers, which reduces the education quality in physicsmathematics classes. Due to the above, a number of operations at laboratory practicums are conducted using digital and analog measuring instruments. Measuring results are introduced manually into Excel tables prepared on PCs. Students draft formulas in Excel spreadsheet tables in order to process data, obtain experiment results and estimate inaccuracies; they plot experimental graphic curves taking into account these inaccuracies. Shaping these general competences while working at practicums is very important so that students may successfully perform at the final examination (Unified State Exam), it is also vital for their further studies at technical and physics universities.

Along with computerized sensors and tablets, at laboratory practicums we use computers with "Electronic oscilloscope" software installed. As a result, such computer becomes a comprehensive digital device, which in real time measures voltage, amperage, oscillation frequency, time and other physical quantities. Students working with electronic oscilloscope do not only measure parameters of physical quantities, but also display all kinds of graphic curves: phase relations of voltage and amperage, oscilloscope records of voltage across a rectifier and electromagnetic oscillations of acoustic frequency. The information may be displayed on a large screen, saved in a convenient format, edited and shared.

Thus, at laboratory practicums the computer performs the functions of a multi-purpose device: it measures physical quantities, acts as a tool for obtaining, visualizing, storing, processing, formalizing and transmitting data. Most time of the lesson students work on their own, using the computer as an education tool, which is managed by the teachers. An important aspect of such education process is the fact that students actively use the computer not as a toy or a screen, but as a work instrument, knowledge of which will help them in their further studies, future work and in daily life.

The use of computerized information technologies at laboratory practicums creates didactically active environment, which encourages cognitive and mental activity of students, makes them ready for active creative activity in information-oriented society, which means that in general it improves education quality.

While organizing practicums we have discovered that a large reserve for implementing the activity approach involves inter-subject relations with information technology. That resulted into a new concept of practicums aimed at analyzing physical processes on the basis of computerized modeling. Such practicums are held taking time allotted for studying information technologies and enable teachers to solve important methodological problems: familiarize students with modeling as a perception method, raise their interest to studying physics and information technology, informally teach them programming and instruct how to use it in practice.

Studying physical processes in computer environment assists students to develop intellectually, master new mindsets, enables them to create their own program products. An undeniable advantage of computerized models is a possibility to visualize a physical process (in the form of motion trajectories, dynamic charts, lines of force, etc).

Practicums aimed at analyzing physical processes on the basis of computerized modeling in subject-oriented classes play an important role: students create a computer model of a physical process and then work with such model $[4,5]$. 
Main peculiarities of practicums aimed at analyzing physical processes on the basis of computerized modeling include:

1. Students fix the goals of research on their own.

2. Students apply modeling as a method of scientific perception of nature in practice.

3. Students are not given instructions on how to carry out the works.

4. Students create computer programs for studying physical processes.

5. Students conduct computer experiments with a model in active mode; they watch specific physical processes, obtain new information on this process.

Practicums aimed at analyzing physical processes on the basis of computerized modeling should be held after holding a correspondent laboratory practicum. Students receive an impetus for intensive development in education process while creating their own ideal models, studying their properties, working with these models as with real things, proving experimentally the correctness of their theoretical assumptions, inventing their own experiments and implementing them in practice.

While holding practicums aimed at analyzing physical processes on the basis of computerized modeling certain stages shall be followed:

1. Fixing a goal based on studying actual physical process (e.g. reveal dependency of current amplitude in series-tuned circuit from alternative current frequency).

2. Shaping a mathematical model by choosing necessary physical quantities, drawing up a set of equations based on physical laws required for process examination, setting equation parameters and other limitations to reach a unique solution.

3. Solution of equation systems and determining the number of fixed and variable parameters and their range of variation.

4. Developing a solution algorithm: usually we use parameter calculation algorithm according to certain equations, obtained by solving the initial system of equations; in some cases we use numerical algorithm for solving the system of equations.

5. Program developing and debugging. Computer models are developed in Visual Basic, Pascal or Excel, depending on the capabilities of the students. The attention shall be paid to the fact that the minimum number of parameters is entered from the keyboard so that the result of program working complies with the fixed goal as much as possible. Program debugging.

6. Computer experiment and results analyzing. The experiments shall be performed multiple times with different parameters, the results are analyzed, summarized and saved, experimental error shall be also evaluated. The results and conclusions are compared with the results of an actual experiment.

It should be noted that the implementation of computer studies of physical processes after a real physical experiment is justified because in the classroom and at laboratory practicums, high school students perform an experimental setup using real devices, and in the course of practicums aimed at analyzing physical processes on the basis of computerized modeling the debugged program acts as an experimental setup of physical processes. Thus, a correspondence between the virtual and the real experiment is made. This correspondence leads to forming students' understanding of a real physical basis of any virtual process, which is important for modern world.

While at practicums aimed at analyzing physical processes on the basis of computerized modeling students create their own programs by using scientific perception method, by modeling physical processes and carrying out computer experiments. All this contributes to the high quality conscious assimilation of physics material, since the ability to model under teacher's supervision certain aspects of reality and understanding of the laws of their structure is a general principle of knowledge assimilation.

Laboratory practicums and practicums aimed at analyzing physical processes on the basis of computerized modeling, except for the above mentioned functions, solve another important problem of modern education: experimental works act as a core for a large amount of tasks, including those in the Unified State Exam. Students independently research the phenomenon, calculate the required physical quantities to construct graphic dependencies based on the results of laboratory and computer experiments, prepare and present the results of their work to the teacher; students work out and learn this material not formally, but consciously, which allows them to successfully solve physics problems. Problem solving is trained at such practicums.

A problem-solving practicum, along with general idea of activity approach, performs an important function: to put knowledge into practice based on students' mental activity. Problem solving is an important procedure to obtain information on student's learning activity and its results. This is a student-teacher feedback. Each class represents a group of students with various ability levels, study motivations, possibilities and life goals. Due to the above, problem solving system shall be differentiated and have a number of difficulty levels.

In order to hold a problem-solving practicum in subject-oriented classes it is recommended to use a system of three-level problems in different versions. The versions shall be of equal difficulty. The levels shall differentiate by their difficulty: basic, advanced and proficient.

Problem-solving practicums have certain distinctive features:

1. Students choose the difficulty level of problems on their own.

2. Students change the difficulty level while solving the problems.

3. Computerized modeling promotes students to see actual processes in problems, separate essential properties, which helps students to solve problems of more complicated levels.

Problem-solving practicums consist of a sequence of lessons: a lesson for problem analysis conducted by both a teacher and students; a practicum for problem solving with the help of the teacher and using a textbook; a practicum for independent problem solving, a test. All practicums shall use information technologies in 
different forms. Let us consider "Study Guide for an Interactive Whiteboard" [6] in detail.

"Study Guide for an Interactive Whiteboard" informational project was created in 2007; it is developing at present, and enjoys the support of pedagogical community. It is a creative, long-term, interdisciplinary and collective project, consisting of many individual projects. The project brings together the activities of teachers and students. Educational computer manuals are created to solve graphic and pictorial physics problems for students of 9-11grades: "Natural Forces", "Thermodynamics", "Direct Current", "Geometrical Optics", "Basic and Unified State Exams". Each of them consists of 50 various dynamic pictorial problems, which are solved using interactive white boards (tablets). Problem solutions can be shown on the board as per request. The students can compare their own solutions with the original right away, make conclusions, and correct the mistakes. For example, "Direct Current" guide comprises problems in calculating current circuit resistance using equivalentcircuit concept and problems in calculating circuit constants using Kirchhoff's laws.

Study guides are Microsoft Office PowerPoint-made presentations prepared by us. All the necessary pictures, photos are scanned or constructed using Microsoft Word, PowerPoint, StarBoard or other software. Each slide layout consists of questions, clues and answers in the form of pictures. The next step is setting the sequence of showing up slide elements: the question, the clue, and the answer (every element shows up on a mouse click). It should be noted that the answer has multiple solutions. As per students' request the phased mode of elements show-up has been introduced.

Once created, the study guide can be used many times in full or in part. It can be used as an enduring simulator. Study guides in current circuit calculations and imaging with the use of lenses are the most popular among students.

"Study Guides for an Interactive White Board" are efficient in explaining the algorithms of new types of problems, in checking home- and self guided works, in acting as electronic task. Organization of education process with the use of study guides for an interactive white board allows including every student into education process as an active member. Students perform different tasks with interest; they can see analysis of the answers right after the solution.

Students may create new slides with problems on their own, thus enlarging the database of Physics Study Guides for an Interactive White Board. As a result, the idea of creating their own interactive study guides has expanded into other school subjects, such as Geometry and Russian.

Is it necessary nowadays to create one's own electronic study guides, when any information can easily be found on the Internet? We believe it is. Every teacher has his own database with study guides of different difficulty levels. Students find and solve their own interesting problems. These problems provide the basis for computer study guides. Making their own computer study guides promotes students to use cognitive activity, create not complicated, but their own information product, which can be used by many students and classes in the future.

Even in the 18th century Lessing wrote: "Think wrongly, if you please, but in all cases think for yourself". In the 21 st century these words did not become obsolete. Thus, the school leaver will not have just a diploma, but will be a "man of thought and study", a subject of study, as he will have to study, think, approach the world in a creative way all his life.

Studying information technologies helps students to adapt themselves to modern society, fulfill their potential in information-extensive world, which means improving the education quality.

The work is done under the guidance of A.A. Fadeeva, Ed.D., professor.

\section{References}

1. Federal State Education Standards for secondary general education, available online: URL http://minobrnauki.rf >Documents> (accessed on: 03.03.16) [In Rus]

2. L.V. Tishchenko, Physics at school, 6, 54-62 (2011)

3. L.V. Tishchenko, Educational physics experiment. Modern technologies: 7 - 11 grades (Ventana-Graph, Moscow, 2015) [In Rus]

4. L.V. Tishchenko, Physics practicum with the use of computerized modeling, 4, 50 - 51 (2010) [In Rus]

5. L.V. Tishchenko, Physics. First day of school year, 18, 5-9 (2007) [In Rus]

6. L.V. Tishchenko, Physics. First day of school year, 17, 10 - 12, (2009) [In Rus] 